This document is the Accepted Manuscript version of a Published Work that appeared in final form in J. Phys.

Chem. C, copyright $(\subseteq)$ American Chemical Society after peer review and technical editing by the publisher. To access the final edited and published work see http://pubs.acs.org/doi/pdf/10.1021/acs.jpcc.6b09880.

\title{
How Is the Surface Tension of Various Liquids Distributed along the Interface Normal?
}

\author{
Marcello Sega ${ }^{1}$, Balázs Fábián, ${ }^{2,3}$ George Horvai, ${ }^{2,4}$ and Pál \\ Jedlovszky ${ }^{4,5 *}$
}

${ }^{1}$ Computational Physics Group, University of Vienna, Sensengasse 8/9, A-1090 Vienna, Austria

${ }^{2}$ Department of Inorganic and Analytical Chemistry, Budapest University of Technology and Economics, Szt. Gellért tér 4, H-1111 Budapest, Hungary

${ }^{3}$ Institut UTINAM (CNRS UMR 6213), Université Bourgogne Franche-Comté, 16 route de Gray, F-25030 Besançon, France

${ }^{4}$ MTA-BME Research Group of Technical Analytical Chemistry, Szt. Gellért tér 4, H-1111 Budapest, Hungary

${ }^{5}$ Department of Chemistry, Eszterházy Károly University, Leányka utca 6, $H$ 3300 Eger, Hungary

Running title: Surface Tension Distribution at Liquid Surfaces

*Electronic mail: jedlovszky.pal@uni-eszterhazy.hu 


\section{$\underline{\text { Abstract }}$}

The tangential pressure profile has been calculated across the liquid-vapor interface of five molecular liquids, i.e., $\mathrm{CCl}_{4}$, acetone, acetonitrile, methanol, and water in molecular dynamics simulations using a recently developed method. Since the value of the surface tension is directly related to the integral of this profile, the obtained results can be interpreted in terms of the distribution of the surface tension along the interface normal, both as a function of distance, either from the Gibbs dividing surface or from the capillary wave corrugated real, intrinsic liquid surface, and also in a layerwise manner. The obtained results show that the surface tension is distributed in a 1-2 nm wide range along the interface normal, and at least $85 \%$ of its value comes from the first molecular layer of the liquid in every case. The remaining, roughly $10 \%$ contribution comes from the second layer, with the exception of methanol, in which the entire surface tension can be accounted for by the first molecular layer. Contributions of the third and subsequent molecular layers are found to be already negligible in every case. 


\section{Introduction}

Surface tension, the intensive counterpart of surface area, is a key quantity in colloid and interface science. At the molecular level, surface tension originates from the fact that particles at the surface of their phase do not have as many and/or as strong attractive interactions with their neighbors in the opposite phase as with those within their own phase. In the particular case of the liquid-vapor interface, far from the critical point, particles that are at the surface practically lack any kind of interactions with the neighboring vapor phase. Evidently, this lack or loss of attractive interactions from the direction of the opposite phase affects not only the particles that are located right at the surface of their phase; particles in the second, third etc .molecular layers also miss some interactions from their outer coordination shells. Having this in mind, the following question can be raised immediately: how do the subsequent subsurface layers contribute to the surface tension of a liquid, in other words, how is the surface tension distributed along the surface normal axis. Considering that surface tension originates from the lack of attractive interactions, the answer to this question can be expected to depend sensibly on the particular intermolecular interactions that characterize the given liquid phase.

Although the above question concerning the distribution of the surface tension is related to the fundamental physical concept laying behind the phenomenon of surface tension, addressing it in a quantitative way is severely hindered by various difficulties. Clearly, experimental investigation of the problem would be extremely challenging, as in this case the average interaction energy of molecules forming the subsequent subsurface layers is needed to be selectively measured. Computer simulations could provide a useful alternative of experiments in this respect, as they can provide a molecular level insight into the structure and interactions of, at least, a suitably chosen model of the system to be studied. However, even in a computer simulation investigation of this problem one has to face several difficulties.

The first such difficulty is that fluid interfaces are corrugated, on the molecular length scale, by capillary waves, which make the determination of the full list of molecules located right at the boundary of their phase a task that is far from being trivial. For this purpose, a number of different methods that are able to locate the real, capillary wave corrugated,

intrinsic surface of a fluid phase have been proposed in the past decade. ${ }^{1-8}$ Among these methods the Identification of the Truly Interfacial Molecules (ITIM) $)^{5}$ turned out to be an 
excellent compromise between computational cost and accuracy. ${ }^{7}$ In an ITIM analysis the molecules of the surface layer are detected by moving a probe sphere of a given radius along a set of test lines from the bulk opposite phase towards the interface in direction parallel with the macroscopic surface normal. Once the first molecule of the phase of interest is touched by the probe it is marked as being interfacial, and the probe starts to move along the next test line. When all the test lines have been considered, the full list of the truly interfacial molecules is identified. ${ }^{5}$ Having the full list of the surface molecules determined, a continuous geometric covering surface of the corresponding phase can be constructed by extrapolating from the positions of the surface particles. ${ }^{9}$ Then, profiles of various physical quantities can be calculated as a function of the distance from this geometric covering surface. ${ }^{9,10}$ Furthermore, by disregarding the set of molecules that constitute the surface layer, and repeating the entire ITIM algorithm, the molecules forming the second (and in subsequent similar steps the third, etc.) molecular layers beneath the liquid surface can also be identified. ${ }^{5,10}$ This provides an alternative way of characterizing thermodynamic quantities at interfaces, namely, by their distribution among the subsequent subsurface molecular layers. ${ }^{10}$

The surface tension, $\gamma$, can simply be calculated at macroscopically planar interfaces as the integral of the difference between the normal and tangential pressure components $\left(p_{\mathrm{N}}\right.$ and $p_{\mathrm{T}}$, respectively) across the interface: ${ }^{11}$

$$
\gamma=\frac{1}{2} \int_{-\infty}^{\infty}\left(p_{\mathrm{N}}-p_{\mathrm{T}}(X)\right) \mathrm{d} X,
$$

where $X$ is the interface normal axis, and the factor of 2 in the denominator accounts for the presence of two interfaces in the basic simulation box. Since mechanical stability of the system requires the normal pressure component, $p_{\mathrm{N}}$, to be constant along the interface normal axis, the calculation of the surface tension profile is equivalent with that of the tangential pressure component. However, the calculation of the tangential pressure profile is hindered by the fact that it requires the localization of a quantity (i.e., the pressure) that is inherently nonlocal. Namely, the pressure contribution corresponding to the interaction of a given particle pair can be calculated as a contour integral along an open path connecting the two particles. ${ }^{12}$ It was shown, however, that several particular choices of the integration contour, such as the Irving-Kirkwood ${ }^{13}$ or the Harasima ${ }^{14}$ path yield comparable profiles of the tangential pressure. ${ }^{15}$ The use of the Harasima path has several additional advantages. First, unlike the Irving-Kirkwood path, it can be used even if the potential energy of the system is not pairwise additive. $^{15}$ The importance of this fact in computer simulations becomes evident when 
considering that even if the intermolecular potential function is pairwise additive, the long range correction of the electrostatic interaction is usually not. In particular, the reciprocal space term occurring in the Ewald summation method ${ }^{16-18}$ and in its particle mesh variants ${ }^{19,20}$ introduces a non-pairwise additive term in the potential. The other advantage of using the Harasima path is that in this way the tangential pressure can be distributed among the individual particles, i.e., a given pressure contribution can be assigned to each of the individual particles, which makes the pressure profile calculation computationally feasible and efficient. ${ }^{10}$ Recently we have demonstrated how the tangential pressure contribution of the long range part of the electrostatic interaction can be taken into account when the computationally very efficient Particle Mesh Ewald (PME) method ${ }^{19}$ or its smooth particle variant ${ }^{20}$ is used in combination with the Harasima integration path. ${ }^{21}$

In this paper we present the calculation of the surface tension profile of five different molecular liquids, i.e., carbon tetrachloride, acetone, acetonitrile, methanol, and water, along the normal axis of their liquid-vapor interface on the basis of molecular dynamics computer simulations. This set of molecular liquids has been chosen to cover a broad range of intermolecular interactions. Namely, $\mathrm{CCl}_{4}$ is characterized solely by van der Waals interaction; acetone and acetonitrile are strongly dipolar, yet aprotic (i.e., non-hydrogen bonding) liquids, methanol consists of small, isolated hydrogen bonded clusters of the molecules, ${ }^{22}$ whereas in liquid water the molecules form a space-filling, percolating hydrogen bonding network. ${ }^{23,24}$ The surface tension profile is determined both relative to the capillary wave-corrugated, intrinsic liquid surface (intrinsic profile), and relative to the center-of-mass of the liquid phase (or, equivalently, to the Gibbs dividing surface between the two phases, non-intrinsic profile). In addition to the entire profiles, the contributions given by the first five molecular layers to them are also calculated in every case. Furthermore, the surface tension contribution is determined also in a layerwise manner. ${ }^{10}$ The obtained distribution of the surface tension contributions along the surface normal axis is discussed in connection with the intermolecular interactions acting in the different liquid systems.

The paper is organized as follows. In section 2 details of the calculations performed, including molecular dynamics simulations, ITIM analyses and tangential pressure / surface tension profile calculations are given. The obtained results are presented and discussed in detail in section 3. Finally, in section 4 the main conclusions of this study are summarized. 


\section{Computational Details}

2.1. Molecular Dynamics Simulations. Molecular dynamics simulations of the vapor- liquid interface of five neat molecular liquids, namely $\mathrm{CCl}_{4}$, acetone, acetonitrile, methanol, and water have been performed in the canonical $(N, V, T)$ ensemble at the temperature $T=280 \mathrm{~K}$, with the exception of water, for which $T=300 \mathrm{~K}$ was used. The schematic structure of these molecules is illustrated in Figure 1. The set of molecules considered has been selected in such a way that the systems studied cover a broad range of intermolecular interactions, from van der Waals $\left(\mathrm{CCl}_{4}\right)$ through dipolar aprotic (acetone and acetonitrile) to non-network forming (methanol) and network-forming (water) hydrogen bonding liquids. The length of the $Y$ and $Z$ edges of the rectangular basic simulation box have both been set to $50 \AA$ for all simulations, while that of the direction normal to the interface, $X$, has been set in the range from 300 to $500 \AA$, depending on the molecular liquid, in order to let the vapor phase be thick enough in every case to separate well the two liquid-vapor interfaces present in the basic box.

$\mathrm{CCl}_{4}$ and acetone molecules have been described by the OPLS ${ }^{25}$ and $\mathrm{TraPPE}^{26}$ potential models, respectively; acetonitrile has been modeled by the potential of Böhm et al., ${ }^{27}$ methanol by that of Walser et $a{ }^{28}{ }^{28}$ whereas water molecules have been described by the threesite $\mathrm{SPC} / \mathrm{E}^{29}$ potential model. To confirm that the results are not depending on the particular potential model chosen we have repeated the calculations concerning water using the $\mathrm{SPC}^{30}$ and TIP3P ${ }^{31}$ models instead of SPC/E, which have left all of our conclusions essentially unchanged. The methyl groups of the acetone and methanol molecules have been treated as united atoms, whereas the acetonitrile model used treats every methyl hydrogen atom separately. All molecular models used are rigid; the geometry of the molecules has been kept unchanged in the simulations by means of the SHAKE algorithm. ${ }^{32}$ The potential models used are all pairwise additive; the intermolecular interaction energy of a molecule pair has been calculated as the sum of the Lennard-Jones and Coulombic interactions of all pairs of their respective interaction sites. The Lennard-Jones distance and energy parameters, $\sigma$ and $\varepsilon$, respectively, and fractional charges, $q$, corresponding to the individual interaction sites of the molecular models used are summarized in Table 1. The Lennard-Jones interaction of unlike pairs of interaction sites has been calculated according to the Lorentz-Berthelot rule. ${ }^{18}$ All interactions have been truncated to zero beyond the cut-off distance of $10 \AA$. The long range part of the electrostatic interaction has been taken into account by the Particle Mesh Ewald 
(PME) method in its smooth variant. ${ }^{20}$ It should be pointed out that here we did not take into account the long-range part of the dispersion forces. To test the importance of this point we have repeated the calculations concerning $\mathrm{CCl}_{4}$ (i.e., the system on which this correction is expected to have the largest impact) using the interaction cut-off value of $15 \AA$. Although the change of the cut-off value affected strongly the actual value of the surface tension as well as of the liquid density, as expected, ${ }^{33,34}$ it left all of our conclusions regarding the shape of the pressure profiles and distribution of the surface tension unchanged.

The simulations have been performed using an in-house modified version of the GROMACS 5.1 molecular dynamics simulation package ${ }^{35}$ that calculates also the pressure contribution of each particle. ${ }^{36}$ The equations of motion have been integrated using the leapfrog algorithm and time steps of $1 \mathrm{fs}$. The temperature of the systems has been controlled by means of the Nosé-Hoover thermostat ${ }^{37,38}$ with a time constant of 0.1 ps. At the beginning of each simulation, the required number of molecules were placed in a basic box, the $Y$ and $Z$ edge lengths of which had already set to their final values, while the length of the $X$ edge was chosen in such a way that the density of the system roughly corresponded to the liquid phase density. After proper energy minimization the systems were equilibrated for at least $0.5 \mathrm{~ns}$ on the isothermal-isobaric $(N, p, T)$ ensemble at 1 bar using the Parrinello-Rahman barostat. ${ }^{39}$ The liquid-vapor interfaces were then created by enlarging the $X$ edge of the basic box to its final value. The interfacial systems were further equilibrated for at least $5 \mathrm{~ns}$. Finally, $20 \mathrm{~ns}$ long equilibrium trajectories have been generated for calculating the surface tension profiles.

2.2. Detection of the Intrinsic Liquid Surface by the ITIM Method. The intrinsic surfaces of the simulated liquid phases have been determined using the ITIM method. ${ }^{5}$ First, a distinction had to be made between molecules that form the liquid phase and those that entered into the vapor phase. For this, a cluster analysis algorithm ${ }^{40}$ has been applied. Thus, we have defined two neighboring molecules to be in "contact" with each other if the distance of any of their two atoms is smaller than a pre-defined cut-off value. Having this definition, we have determined all the clusters formed by the molecules in the system, and have found the largest of them. Clusters have been defined as assemblies of molecules any two of which are connected via an intact chain of molecule pairs that are in contact with each other. We have regarded the largest of these clusters as the liquid phase itself, while the smaller clusters (including the isolated molecules) have been considered as part of the vapor phase. ${ }^{40,41}$ The cut-off values used in defining the contact position of two neighboring molecules have been set to $8.0,5.0,3.6,5.8$, and $3.5 \AA$ for $\mathrm{CCl}_{4}$, acetone, acetonitrile, methanol, and water, 
respectively, these values being the smallest of the first minimum positions of the partial radial distribution functions of the corresponding liquid, excluding the ones involving explicit hydrogen atoms. The results of the subsequent analyses turned out to be rather insensitive to the particular choice of these cut-off values.

For each configuration, after determining the liquid phase, we proceeded to compute the set of interfacial atoms using the ITIM algorithm ${ }^{5}$ as described above, using a probe sphere with a radius $R_{\mathrm{p}}$ of $2 \AA$. Contact position of the probe sphere with a given atom has been defined as their center to center distance being equal to the sum of the respective radii; the diameter of the atoms has been estimated by their Lennard-Jones distance parameter, $\sigma$ (see Table 1). It should be emphasized that the surface layer has been defined on a molecular basis: once the probe sphere touches an atom the entire molecule to which this atom belongs is considered as part of the layer. Test lines have been arranged on a square grid, neighboring lines being separated by $0.5 \AA$ from each other. The entire procedure of detecting the topmost layer of the liquid phase has been repeated five times by disregarding the molecules that belong to one of the previously identified layers. In this way, the molecules forming the first five subsequent layers of the liquid phase have been identified separately. These layers are illustrated in Figure 2, showing an equilibrium snapshot of each of the five systems considered. Finally, all results have been averaged over the two liquid-vapor interfaces present in the basic box.

2.3. Calculation of the Surface Tension Profile. The global pressure tensor can, in general, be expressed as a sum of the ideal gas contribution (kinetic term) and an excess contribution (the virial term, $\Xi$ ):

$$
p_{\mu v}=\frac{2}{V}\left(\frac{1}{2} \sum_{i} m_{i} v_{i}^{\mu} v_{i}^{\nu}-\Xi_{\mu v}\right) .
$$

While the local expression for the kinetic contribution is trivial, that of the virial, $\Xi(\mathbf{r})$, is not. For pairwise additive forces, $f_{i j}$, $\Xi(\mathbf{r})$ can be written as an integral along an open path, $C_{i j}$, parametrized by the vector $\mathbf{s}$, connecting each pair $(i, j)$ of atoms:

$$
\Xi_{\mu v}(\mathbf{r})=-\frac{1}{2}\left\langle\sum_{i j} f_{i j}^{\mu} \int_{C_{i j}} d s^{v} \delta(\mathbf{r}-\mathbf{s})\right\rangle .
$$


In these equations Latin indices ( $i$ and $j$ ) identify particles, while the Greek indices ( $\mu$ and $v$ ) identify spatial direction. This expression depends on the choice of the integration path, an unavoidable consequence of the virial being intrinsically a non-local quantity, ${ }^{12}$ but yields qualitatively similar results ${ }^{15}$ for two of the commonly used paths, namely the IrvingKirkwood $^{13}$ and the Harasima ${ }^{14}$ ones. The latter is the path of choice for our simulations, as it concentrates the local tangential pressure contributions on the atomic positions, and allows calculating the local pressure contribution also for the non-pairwise additive (i.e., reciprocal space) part of the Ewald sum and its particle-mesh variants. The reciprocal space contribution of the Ewald sum can be expressed as a sum over the contributions of the individual particles: ${ }^{15,21}$

$$
\Xi_{\mu \nu}^{\mathrm{rec}, i}(\mathbf{r})=\frac{q_{i}}{V} \sum_{m \neq 0} \operatorname{Re}\left[\frac{\exp \left(-2 \pi \mathbf{m} \cdot \mathbf{r}_{i}\right)}{2 \pi V} S(\mathbf{m}) f\left(\mathbf{m}^{2}\right) g_{\mu \nu}(\mathbf{m})\right]
$$

where the sum extends over the reciprocal space vectors, $\mathbf{m}$, and depends on the charge structure factor:

$$
S(\mathbf{m})=\sum_{i} q_{i} \exp \left(2 \pi \mathbf{m} \cdot \mathbf{r}_{i}\right)
$$

as well as on the functions

$$
f(\mathbf{m})=\exp \left\lfloor-\pi^{2} \mathbf{m}^{2}\left(k_{\mathrm{B}} T\right)^{2}\right\rfloor
$$

and

$$
g_{\mu v}(\mathbf{m})=\delta_{\mu v}-2 \frac{1+\pi^{2} \mathbf{m}^{2}\left(k_{\mathrm{B}} T\right)^{2}}{\mathbf{m}^{2}} \mathbf{m}_{\mu} \mathbf{m}_{v}
$$

The mesh-based variant distributes the virial contribution on the mesh nodes at positions $\mathbf{r}_{p}$ $\operatorname{as}^{21}$

$$
\widetilde{\Xi}_{\mu \mu}^{\mathrm{rec}, i}\left(\mathbf{r}_{p}\right)=\mathrm{FFT}^{-1}\left\{B(\mathbf{m}) F F T[\tilde{\rho}] f\left(\mathbf{m}^{2}\right) g_{\mu \mu}(\mathbf{m})\right\},
$$

where the charge distribution on the mesh of spacing $h$ using the assignment function $W$ is

$$
\tilde{\rho}\left(\mathbf{r}_{p}\right)=\frac{1}{h} \sum_{i} W\left(\mathbf{r}_{p}-\mathbf{r}_{i}\right) q_{i}
$$


and $B(\mathbf{m})$ depends on the specific interpolation scheme (e.g., b-splines). The virial contribution can be interpolated back to obtain the contributions of the particles as

$$
\Xi_{\mu \mu}^{\mathrm{rec}, i}=\frac{q_{i}}{V} \sum_{r_{p}} \widetilde{\Xi}_{\mu \mu}^{\mathrm{rec}, i}\left(r_{p}\right) W\left(r_{i}-r_{p}\right) .
$$

Note that the Harasima path allows the calculation only of the tangential components of the local virial tensor, so that the above formulas for the Ewald and mesh-Ewald contributions are valid only for $\mu$ and $v$ equal to either $Y$ or $Z$. This is, in general, not a problem, as the normal component of the virial tensor has to be constant to ensure mechanical stability of the system, and can thus be simply calculated from the global virial.

All other terms of the virial (i.e., the ones corresponding to the real space part of the Ewald sum, exclusions, and the SHAKE algorithm) can be trivially decomposed to the respective particle contributions. It is therefore possible to write in our geometry the tangential pressure contribution of each particle as

$$
p_{\mathrm{T}}^{i}=\frac{1}{V}\left[\frac{1}{2} m_{i}\left(v^{Y} v^{Y}+v^{Z} v^{Z}\right)-\left(\Xi_{Y Y}^{i}+\Xi_{Z Z}^{i}\right)\right]
$$

Once the particle contributions have been determined, the tangential pressure profile can readily be calculated both in its non-intrinsic and intrinsic variants:

$$
p_{\mathrm{T}}(X)=\frac{V}{A}\left\langle\sum_{i} p_{\mathrm{T}}^{i} \delta\left(X-X_{i}+X_{\mathrm{cm}}\right)\right\rangle
$$

and

$$
p_{\mathrm{T}}\left(X_{\mathrm{intr}}\right)=\frac{V}{A}\left\langle\sum_{i} p_{\mathrm{T}}^{i} \delta\left(X_{\mathrm{intr}}-X_{i}+\xi\right)\right\rangle
$$

where $X_{\mathrm{cm}}$ is the position of the center of mass of the liquid slab along the surface normal, and $\xi$ is the local position of the interface along the same axis.

\section{Results and Discussion}

The surface tension values obtained by integrating the $p_{\mathrm{T}}(X)$ curves, as described in the previous subsection, turned out to be $14.6,15.9,20.8,17.3$, and $58.5 \mathrm{mN} / \mathrm{m}$, with the error 
bars never exceeding $0.2 \mathrm{mN} / \mathrm{m}$, for $\mathrm{CCl}_{4}$, acetone, acetonitrile, methanol, and water, respectively. As a consistency check, we have repeated this calculation using the intrinsic pressure profiles, $p_{\mathrm{T}}\left(X_{\text {intr }}\right)$ (where $X_{\text {intr }}$ denotes the distance from the intrinsic surface along the macroscopic surface normal axis, $X$ ), and calculated $\gamma$ also in the conventional way, using the average value of $p_{\mathrm{T}}$ as obtained from the global pressure tensor, without distributing it among the particles. The surface tension values obtained in these three different ways indeed turned out to be identical in every case up to the numerical precision of the calculations.

3.1. Non-Intrinsic Tangential Pressure Profiles. The non-intrinsic profiles of the tangential pressure, $p_{\mathrm{T}}(X)$, are shown in Figure 3 along with the contributions given by the first five molecular layers beneath the liquid surface, as obtained for the five liquids considered. In these profiles, the $X=0 \AA$ value corresponds to the center of mass position of the liquid slab, and the vapor phase is located at $X$ values larger than what corresponds to the position of the interface. The variation of the position of the interface along the $X$ axis from system to system simply reflects the different densities of the different liquids. The interfacial part of the $p_{\mathrm{T}}(X)$ profiles and its layerwise contributions are shown on magnified scales in the insets of Fig. 3.

As is seen, the tangential pressure profile shows a deep minimum at the liquid side of the interface, whereas the rest of the profile is practically featureless in every case. This negative peak of the $p_{\mathrm{T}}(X)$ profiles covers an about 10-20 $\AA$ wide $X$ range, being the narrowest for water $(\sim 7 \AA)$ and broadest for $\mathrm{CCl}_{4}(\sim 19 \AA)$. This finding indicates that the surface tension is indeed located in a narrow, 1-2 $\mathrm{nm}$ wide region of the liquid at the surface. This width is larger by roughly a factor of three than the molecular size. However, due to the presence of capillary fluctuations, from the total profile it is not possible to draw any conclusion in how many molecular layers the surface tension is concentrated. Still, differences in the width of this region reflect essentially the different size of the molecules constituting the liquid phases. However, the range of this region in water is particularly narrow, and this cannot be accounted for simply by the small size of the water molecules. Rather, this narrowness is also due to the unusually strong (H-bonding) intermolecular interaction in water, which leads to rather close packing of the molecules and high density of the liquid.

The minimum of this negative peak of $p_{\mathrm{T}}(X)$ corresponds to rather large negative tangential pressure values, being around -200 - -300 bar in every case, with the exception of water, where this value is close to -2000 bar. Since the value of the surface tension, $\gamma$, is 
related to the integral of this peak (see eq 1), it is not surprising that in liquids of similar surface tension values the depth of this minimum is also similar. However, the surface tension of the water model used here is only 3-4 times larger than that of the models of the other liquids considered, while the minimum of the $p_{\mathrm{T}}(X)$ profile is an order of magnitude deeper. This is the consequence of the aforementioned exceptional narrowness of the negative $p_{\mathrm{T}}(X)$ peak in water: narrow peaks must be larger in magnitude to result in the same integral value.

By looking at the decomposition of the pressure profiles into the contributions coming from different layers, also shown in Fig. 3, it is seen that that the large negative peak of the $p_{\mathrm{T}}(X)$ profile is largely accounted for by the contribution of the first single molecular layer beneath the liquid surface in every case. In other words, the vast majority of the surface tension is distributed within the $X$ range in which the first molecular layer is located. However, the profiles of the contributions of the subsequent molecular layers are not simply zero along the entire interface normal axis, instead, they typically exhibit a negative loop followed by a positive loop upon going towards the interface. In a few cases (e.g., the second layer of $\mathrm{CCl}_{4}$, acetone, or acetonitrile) the negative loop is seen to be larger in magnitude than the subsequent one, indicating the non-negligible contribution of also these layers to the surface tension. On the other hand, for the majority of the cases the two loops are roughly equal in magnitude, suggesting that these layers do not give considerable contributions to the value of the surface tension. It is also seen that the position of the negative loop of a given layer typically coincides with that of the positive loop of the next molecular layer. As a consequence, the contributions of such two loops corresponding to two subsequent molecular layers largely cancel out each other, making the full $p_{\mathrm{T}}(X)$ profile featureless beneath the $X$ range of the first molecular layer.

The contributions of the second to fifth layers to $p_{\mathrm{T}}(X)$ behave somewhat differently in water in this respect. Thus, here a small negative loop is typically followed by a larger positive, and then again by a small negative loop as going from the bulk liquid phase towards the interface. This "Mexican hat" shaped contribution is probably at least partly due to the fact that the radius of the probe sphere used in the ITIM procedure (i.e., $2 \AA$ ) is somewhat too large for probing the relatively small water molecules. ${ }^{7,42}$ To confirm this, we have repeated the entire analysis for water using a probe sphere of the radius of $1.2 \AA$. In this case, the $p_{\mathrm{T}}(X)$ contributions of the second to fifth layers changed considerably toward the usual (i.e., negative-positive loops) shape, leaving all other findings of us practically unchanged. It is also interesting to note that decreasing the probe sphere radius leaves the contribution of the 
first layer practically unchanged, but broadens and shifts towards the bulk that of the subsequent layers (see Figs S1 and S2 of Supporting Information).

3.2. Intrinsic Tangential Pressure Profiles. The intrinsic profiles of the tangential pressure, $p_{\mathrm{T}}\left(X_{\text {intr }}\right)$, are shown in Figure 4 along with the contributions given by the first five molecular layers beneath the liquid surface, as obtained in the five systems considered. Here the $X_{\text {intr }}=0 \AA$ value corresponds exactly to the position of the intrinsic liquid surface, whereas negative and positive $X_{\text {intr }}$ values correspond to the liquid and vapor phases, respectively.

As it is seen, the intrinsic tangential pressure profiles show considerably more structure than the non-intrinsic ones, although the distance range covering this structured part is typically slightly narrower than that in the case of the non-intrinsic profiles. Also, the profiles of acetonitrile and methanol are somewhat more structured than those of the other systems considered. However, these fine structures are entirely described by contribution of the first molecular layer, and the distances of the peaks in this region are compatible with intramolecular distances, suggesting that this fine structure reflects intramolecular contributions (through the constraints keeping the molecular geometry rigid) rather than intermolecular ones to the pressure profile. The most apparent feature of the $p_{\mathrm{T}}\left(X_{\text {intr }}\right)$ profiles is the Dirac delta-like contribution at $X_{\text {intr }}=0 \AA$. Since the intrinsic liquid surface is defined through a set of interfacial atoms (i.e., the ones that stop the probe sphere in the ITIM procedure), all such atoms are located at exactly $X_{\text {intr }}=0 \AA$. This leads to the appearance of a peak here that is infinitely sharp, yet corresponds to a finite integral. Although the infinite sharpness of this peak is clearly an artifact due to the definition of the intrinsic liquid surface, real physical meaning can be attributed to its (finite) integral. Besides the Dirac delta-like peak at $X_{\text {intr }}=0 \AA$, the $p_{\mathrm{T}}\left(X_{\text {intr }}\right)$ profiles show several oscillations at the liquid side of the intrinsic surface before they decay to a constant value. (At the vapor side they decay rapidly because of the small intermolecular correlation of molecules in the vapor phase.)

It is also apparent from Fig. 4 that the amplitude of the peaks of $p_{\mathrm{T}}\left(X_{\text {intr }}\right)$ at the vicinity of $X_{\text {intr }}=0 \AA$ is typically much larger than that of the main negative peak of the corresponding non-intrinsic profile. Thus, in the case of the non-H-bonding liquids there is a factor of 3-5 between the amplitudes in the two types of profiles, while for methanol the $p_{\mathrm{T}}\left(X_{\mathrm{intr}}\right)$ peak is about an order of magnitude and for water almost two orders of magnitude larger than that of $p_{\mathrm{T}}(X)$.

The majority of the features of the intrinsic tangential pressure profiles are again given by the molecules constituting the first layer beneath the liquid surface. The fact that the first 
layer molecules contribute to $p_{\mathrm{T}}\left(X_{\text {intr }}\right)$ other than just at $X_{\text {intr }}=0 \AA$ is due to the fact that the layers have been defined in terms of molecules, whereas the tangential pressure is distributed among the atoms in the system. In other words, atoms of the surface molecules that are located right at the surface themselves (i.e., those that stop the probe sphere in the ITIM procedure) give rise to the Dirac delta-like peak, while the other atoms belonging to the surface molecules give rise to the contributions at non-zero $X_{\text {intr }}$ values. It should also be noted that the features of the $p_{\mathrm{T}}\left(X_{\mathrm{intr}}\right)$ profiles that correspond to the first molecular layer are of little interest for our purpose, as they simply describe how the pressure is distributed within the molecules.

While the contribution of the first molecular layer already largely describes the entire $p_{\mathrm{T}}\left(X_{\text {intr }}\right)$ profile in every case, the contribution of the second layer is still noticeable in every case. Thus, in $\mathrm{CCl}_{4}$ and acetone the second layer contribution fully accounts for the negative peak at about $X_{\mathrm{intr}}=-7 \AA$ and the positive peak around $X_{\mathrm{intr}}=-3 \AA$, in acetonitrile it describes the waves of $p_{\mathrm{T}}\left(X_{\text {intr }}\right)$ between -10 and $-5 \AA$, and contributes to the negative peak around $-4 \AA$, in methanol it gives rise to the small positive peaks at about -5 and $-3 \AA$, and in water it describes the features of $p_{\mathrm{T}}\left(X_{\mathrm{intr}}\right)$ between about -4 and $-2 \AA$. On the other hand, the contributions of the third to fifth layers to the intrinsic tangential pressure profiles, in a clear contrast to those to the non-intrinsic profiles, are indeed featureless, flat curves.

Finally, it should be noted that the intrinsic tangential pressure profiles show several details that are not seen in the non-intrinsic profiles. Thus, the intrinsic profiles exhibit considerably more features in a considerably narrower distance range than the non-intrinsic ones. Thus, in contrast with the rather complex structure of the intrinsic profiles, the nonintrinsic ones exhibit one single negative peak, the amplitude of which is also much smaller than those of the corresponding intrinsic profiles. All these changes reflect how the real, molecular level structure of the liquid surface is washed out by neglecting the effect of the capillary waves in a non-intrinsic analysis.

3.3. Contributions of the Individual Molecular Layers to the Surface Tension. The net contribution of the subsequent molecular layers beneath the liquid surface to the value of the tangential pressure can be calculated simply by integrating their individual contributions to the tangential pressure profile. Here it is important to notice that it is not possible to decompose the normal pressure component into layer contributions, and therefore, strictly speaking, also the layer contribution to the full surface tension is not accessible, but only its tangential contribution. However, since the normal pressure must be constant along the 
interface normal axis, it is sensible to assume that each layer contributes roughly equally to it, and through it also to the total value of the surface tension. Further, inside the bulk phases the tangential and normal pressure components must be equal to each other and constant along the interface normal axis. It is also clear from Figures 3 and 4 that this constant value is several orders of magnitude smaller than the amplitude of the peaks of the tangential profile (both in the intrinsic and non-intrinsic case). Therefore, apart from the first few molecular layers, the difference of the normal and tangential pressure components (which, in turn, really contributes to the surface tension, see eq. 1) must be zero, whereas in the first few layers the (unknown) normal pressure component must be much smaller than the tangential one, and hence can be neglected. In this sense, the tangential pressure contributions of the individual molecular layers can be interpreted as being practically equal to their full surface tension contributions. Thus, the term of the 'surface tension contribution', when speaking about individual molecular layers, is used and has to be interpreted in the above sense throughout this paper.

The obtained contributions of the first four molecular layers to the surface tension (in percentage) are shown in Figure 5. (The contribution of the fifth layer turned out to be negligible in every case). As a check of consistency, we have done this calculation by integrating both the intrinsic and non-intrinsic pressure profile contributions; the obtained results agreed within $0.1 \%$ in every case. As is seen, the distribution of the surface tension among the molecular layers is rather similar in the different liquids considered, in spite of the fact that they correspond to markedly different intermolecular interactions. Thus, the vast majority, i.e., at least $85 \%$ of the surface tension is concentrated in the first molecular layer; the second layer still gives a noticeable, roughly $10 \%$ contribution to the surface tension, while this contribution is already negligible beyond the second layer in every case. The only exception in this respect is methanol, in which practically the entire surface tension comes from the first molecular layer.

It is also apparent from Fig. 5 that several layers give a negative contribution to the surface tension value. To understand the origin of this finding one has to consider that although the origin of the surface tension is the loss of attractive interaction of molecules being at the boundary of their phase, the surface tension itself describes the free energy cost of molecules being at the surface. More specifically, in order to decrease their energy loss surface molecules can increase their orientational order, both relative to the surface and to each other, which eventually leads the decrease of their entropy. However, while this orientational ordering might mostly or exclusively affect the surface layer itself, the 
corresponding energy gain can also affect the neighboring layers, which can lead to a net free energy decrease (i.e., negative surface tension contribution) in certain layers. This free energy decrease is, however, not supposed to be large; in accordance with our finding that such negative contribution to $\gamma$ never exceeds $1.5 \%$.

Although the general trends in the distribution of the surface tension clearly turn out to be system independent, details of the whole picture still depend on the particular intermolecular interactions acting in the different liquids. Thus, the roughly $10 \%$ contribution of the second layer reflects the lack of attractive interaction of these molecules with several second shell neighbors. In methanol, however, this contribution is completely missing. As it is known, in methanol the surface molecules adopt a rather strong orientational order, preferring the alignment in which the $\mathrm{CH}_{3}$ group sticks straight out to the vapor phase. This orientation allows the surface methanol molecules to maintain all of their possible hydrogen bonds, and therefore to fully preserve their hydrogen bonding structure, ${ }^{43,44}$ which leads to a much lower surface tension value than what could be expected considering the strength of the intermolecular interactions. Since in methanol H-bonding is the dominant interaction, but the vicinity of the interface leaves the H-bonding structure of the molecules unchanged, molecules in the second layer lack only a negligible fraction of their attractive interaction with respect to the bulk phase ones. On the other hand, in water the surface molecules adopt orientations such that they can form as many hydrogen bonds with each other as possible. ${ }^{5,43}$ As a price for that, hydrogen bonding between the first and second layers is, on average, weaker than in the bulk liquid phase.

Finally, it should also be noted that the obtained contributions of the individual molecular layers to the surface tension depend slightly also on the probe sphere radius used in the analysis, although the global picture is insensitive to that. Thus, when using a probe sphere of the radius of $1.2 \AA$ instead of $2 \AA$ for water, the contribution of the first and second layer turns out to be $96.3 \%$ and $3.8 \%$, respectively, while that of the subsequent layers is practically zero. Similarly, when using the $R_{\mathrm{p}}$ value of $1.65 \AA$ instead of $2 \AA$ for methanol, the contribution of the first three layers turns out to be $106.1 \%,-3.5 \%$ and $-1.3 \%$, respectively. The fact that the decrease of the probe sphere radius leads to an increased contribution of the first layer is consistent with the previous finding that it shifts the peaks of the subsequent molecular layers contributions towards the bulk liquid phase (see Figs. S1 and S2 of Supporting Information), and can be explained by the fact that smaller probe spheres can fall deeper (with respect to larger ones) between the molecules, touching molecules located farther from the liquid surface. ${ }^{5}$ 


\section{Summary and Conclusions}

In this paper we have demonstrated how intrinsic surface analysis methods can provide a deep insight into the thermodynamics of fluid surfaces, by calculating the tangential pressure profile across the liquid-vapor interface of five molecular liquids, characterized by markedly different intermolecular interactions. Since the value of the surface tension can be obtained by integrating the tangential pressure profile, this profile provides direct information on how the surface tension is distributed along the interface normal axis.

Our results show a surprising insensitivity of this distribution of the surface tension to the type of intermolecular interactions acting in the liquid phase. Thus, in every case, the vast majority, i.e., at least $85 \%$ of the surface tension comes from the first molecular layer, while, with the exception of methanol, the second layer contributes roughly $10 \%$ to the surface tension, and the contributions of the subsequent molecular layers are negligible. In methanol, on the other hand, the entire surface tension comes practically from the molecules constituting the first layer. Correspondingly, when considering an external (i.e., non-intrinsic) coordinate frame, the surface tension is distributed in a 1-2 nm broad interval along the surface normal, which covers the distance range in which the first molecular layer is located. Considering an intrinsic frame, i.e., calculating the profile relative to the capillary wave corrugated interface itself, the distance range covering non-negligible surface tension contributions is even slightly narrower, and the amplitudes of the peaks of the profile are considerably larger than in the non-intrinsic case. The intrinsic analysis of the contribution of the subsequent molecular layers to the profile also reveals that practically no surface tension contribution comes from the third and subsequent molecular layers. By contrast, in a non-intrinsic treatment this information is obscured by the effect of neglecting the capillary waves in the analysis.

Although the distribution of the surface tension along the surface normal axis turned out to be largely independent from the interactions acting between the molecules in the specific systems, several details of it still reflect the peculiarities of these intermolecular interactions. Thus, in the case of water, characterized by small molecular size, particularly strong interactions and, consequently, particularly close contact of the molecules, the featured part of the surface tension profile is unusually narrow and the amplitudes of its peaks are considerably larger than what could be expected simply from the value of the surface tension. Also, due to the relatively weak interaction between the first two molecular layers beneath the liquid surface, the contribution of the second layer to the surface tension is somewhat larger in 
water than in the other liquids. By contrast, the strong surface orientation of the molecules at the surface of methanol not only efficiently reduces the surface tension, but also eliminates practically the contribution of the second layer.

Supporting Information. Contributions of the first five layers to the non-intrinsic tangential pressure profile in water and methanol are provided, as obtained using probe spheres of different radii. This information is available free of charge via the Internet at http://pubs.acs.org.

Acknowledgements. This work has been supported by the Hungarian NKFIH Foundation under Project No. NKFIH 119732, and by the Action Austria-Hungary Foundation under project No. 93 öu3.

\section{References}

(1) Chacón, E.; Tarazona, P. Intrinsic Profiles beyond the Capillary Wave Theory: A Monte Carlo Study. Phys Rev. Letters 2003, 91, 166103-1-4.

(2) Mezei, M. A New Method for Mapping Macromolecular Topography. J. Mol. Graphics Modell. 2003, 21, 463-472.

(3) Chowdhary, J.; Ladanyi, B. M. Water-Hydrocarbon Interfaces: Effect of Hydrocarbon Branching on Interfacial Structure. J. Phys. Chem. B. 2006, 110, 15442-15453.

(4) Jorge, M.; Cordeiro, M. N. D. S. Intrinsic Structure and Dynamics of the Water/Nitrobenzene Interface. J. Phys. Chem. C. 2007, 111, 17612-17626.

(5) Pártay, L. B.; Hantal, G.; Jedlovszky, P.; Vincze, Á.; Horvai, G. A New Method for Determining the Interfacial Molecules and Characterizing the Surface Roughness in Computer Simulations. Application to the Liquid-Vapor Interface of Water. J. Comp. Chem. 2008, 29, 945-956.

(6) Wilard, A. P.; Chandler, D. Instantaneous Liquid Interfaces. J. Phys. Chem. B. 2010, 114, 1954-1958.

(7) Jorge, M.; Jedlovszky, P.; Cordeiro, M. N. D. S. A Critical Assessment of Methods for the Intrinsic Analysis of Liquid Interfaces. 1. Surface Site Distributions. J. Phys. Chem. C. 2010, 114, 11169-11179. 
(8) Sega, M.; Kantorovich, S.; Jedlovszky, P; Jorge, M. The Generalized Identification of Truly Interfacial Molecules (ITIM) Algorithm for Nonplanar Interfaces. J. Chem. Phys. 2013, 138, 044110-1-10.

(9) Jorge, M.; Hantal, G.; Jedlovszky, P.; Cordeiro, M. N. D. S. A Critical Assessment of Methods for the Intrinsic Analysis of Liquid Interfaces: 2. Density Profiles. J. Phys. Chem. C. 2010, 114, 18656-18663.

(10) Sega, M.; Fábián, B.; Jedlovszky, P. Layer-by-Layer and Intrinsic Analysis of Molecular and Thermodynamic Properties across Soft Interfaces. J. Chem. Phys. 2015, 143, 114709-1-8.

(11) Rowlinson, J. S.; Widom, B. Molecular Theory of Capillarity; Dover Publications: Mineola, 2002, p. 11.

(12) Schofield, P.; Henderson, J. R. Statistical Mechanics of Inhomogeneous Fluids. Proc. R. Soc. Lond. A 1982, 379, 231-246.

(13) Irving, J. H.; Kirkwood, J. G. The Statistical Mechanical Theory of Transport Processes. IV. The Equations of Hydrodynamics. J. Chem. Phys. 1950, 18, 817-829.

(14) Harasima, A. Molecular Theory of Surface Tension. Adv. Chem. Phys. 1958, 1, 203 237.

(15) Sonne, J.; Hansen, F. Y.; Peters, G. H. Methodological Problems in Pressure Profile Calculations for Lipid Bilayers. J. Chem. Phys. 2005, 122, 124903-1-9.

(16) Ewald, P. Die Berechnung Optischer und Elektrostatischer Gitterpotentiale. Ann. Phys. 1921, 369, 253-287.

(17) de Leeuw, S. W.; Perram, J. W.; Smith, E. R. Simulation of Electrostatic Systems in Periodic Boundary Conditions. I. Lattice Sums and Dielectric Constants. Proc. R. Soc. Lond. A 1980, 373, 27-56.

(18) Allen, M. P.; Tildesley, D. J. Computer Simulation of Liquids; Clarendon Press: Oxford, 1987.

(19) Darden, T.; York, D.; Pedersen, L. Particle Mesh Ewald: An N.log(N) Method for Ewald Sums in Large Systems. J. Chem. Phys. 1993, 98, 10089-10092.

(20) Essman, U.; Perera, L.; Berkowitz, M. L.; Darden, T.; Lee, H.; Pedersen, L. G. A Smooth Particle Mesh Ewald Method. J. Chem. Phys. 1995, 103, 8577-8594.

(21) Sega, M.; Fábián, B.; Jedlovszky, P. Pressure Profile Calculation with Particle Mesh Ewald Methods. J. Chem. Theory Comput. 2016, 12, 4509-4515. 
(22) Pálinkás, G.; Hawlicka, E.; Heinzinger, K. A Molecular Dynamics Study of Liquid Methanol with a Flexible Three-Site Model. J. Phys. Chem. 1987, 91, 4334-4341.

(23) Geiger, A.; Stillinger, F. H.; Rahman, A. Aspects of the Percolation Process for Hydrogen-Bond Networks in Water. J. Chem. Phys. 1979, 70, 4185-4193.

(24) Stanley, H. E.; Teixeira, J. Interpretation of the Unusual Behavior of $\mathrm{H}_{2} \mathrm{O}$ and $\mathrm{D}_{2} \mathrm{O}$ at Low Temperatures. Test of a Percolation Model. J. Chem. Phys. 1980, 73, 3404-3422.

(25) Duffy, E. M.; Severance, D. L.; Jorgensen, W. L. Solvent Effects on the Barrier to Isomerization for a Tertiary Amide from $A b$ Initio and Monte Carlo Calculations. $J$. Am. Chem. Soc. 1992, 114, 7535-7542.

(26) Stubbs, J. M.; Potoff, J. J.; Siepmann, J. I. Transferable Potentials for Phase Equilibria. 6. United-Atom Description for Ethers, Glycols, Ketones, and Aldehydes. J. Phys. Chem. B 2004, 108, 17596-17605.

(27) Böhm, H. J.; McDonald, I. R.; Madden, P. A. An Effective Pair Potential for Liquid Acetonitrile. Mol. Phys. 1983, 49, 347-360.

(28) Walser, R.; Mark, A. E.; van Gunsteren, W. F.; Lauterbach , M.; Wipff, G. The Effect of Force-Field Parameters on Properties of Liquids: Parametrization of a Simple Three-Site Model for Methanol. J. Chem. Phys. 2000, 112, 10450-10459.

(29) Berendsen, H. J. C.; Grigera, J. R.; Straatsma, T. The Missing Term in Effective pair Potentials. J. Phys. Chem. 1987, 91, 6269-6271.

(30) Berendsen, H. J. C.; Postma, J. P. M.; van Gunsteren, W. F.; Hermans, J. Interaction Models for Water in Relation to Protein Hydration. In Intermolecular Forces; Pullman, B., Ed.; Reidel: Dordrecht, 1981, p. 331-342.

(31) Jorgensen, W. L.; Chandrashekar, J.; Madura, J. D.; Impey, R.; Klein, M. L. Comparison of Simple Potential Functions for Simulating Liquid Water. J. Chem. Phys. 1983, 79, 926-935.

(32) Ryckaert, J. P.; Ciccotti, G.; Berendsen, H. J. C. Numerical Integration of the Cartesian Equations of Motion of a System With Constraints; Molecular Dynamics of n-Alkanes. J. Comp. Phys. 1977, 23, 327-341.

(33) Trokhymchuk, A.; Alejandre, J. Computer Simulations of Liquid/Vapor Interface in Lennard-Jones Fluids: Some Questions and Answers. J. Chem. Phys. 1999, 111, 85108523.

(34) in 't Veld, P. J.; Ismail, A. E.; Grest, G. S. Application of Ewald Summations to LongRange Dispersion Forces. J. Chem. Phys. 2007, 127, 144711-1-8. 
(35) Pronk, S.; Páll, S.; Schulz, R.; Larsson, P.; Bjelkmar, P.; Apostolov, R.; Shirts, M. R.; Smith, J. C.; Kasson, P. M.; van der Spoel; D., et al. GROMACS 4.5: A HighThroughput and Highly Parallel Open Source Molecular Simulation Toolkit. Bioinformatics 2013, 29, 845-854.

(36) The code is freely available at https://github.com/Marcello-Sega/gromacs/tree/virial/.

(37) Nosé, S. A Molecular Dynamics Method for Simulations in the Canonical Ensemble. Mol. Phys. 1984, 52, 255-268.

(38) Hoover, W. G. Canonical Dynamics: Equilibrium Phase-Space Distributions. Phys. Rev. A 1985, 31, 1695-1697.

(39) Parrinello, M.; Rahman, A. Polymorphic Transitions in Single Crystals: A New Molecular Dynamics Method. J. Appl. Phys. 1981, 52, 7182-7190.

(40) Pártay, L. B.; Horvai, G.; Jedlovszky, P. Temperature and Pressure Dependence of the Properties of the Liquid-Liquid Interface. A Computer Simulation and Identification of the Truly Interfacial Molecules Investigation of the Water-Benzene System. $J$. Phys. Chem. C. 2010, 114, 21681-21693.

(41) Darvas, M.; Jorge, M.; Cordeiro, M. N. D. S.; Kantorovich, S. S.; Sega, M.; Jedlovszky, P. Calculation of the Intrinsic Solvation Free Energy Profile of an Ionic Penetrant Across a Liquid/Liquid Interface with Computer Simulations. J. Phys. Chem. B 2013, 117, 16148-16156.

(42) Sega, M. The Role of a Small-Scale Cutoff in Determining Molecular Layers at Fluid Interfaces. Phys. Chem. Chem. Phys. 2016, 18, 23354-23357.

(43) Pártay, L. B.; Jedlovszky, P.; Vincze, Á.; Horvai, G. Properties of Free Surface of Water-Methanol Mixtures. Analysis of the Truly Interfacial Molecular Layer in Computer Simulation. J. Phys. Chem. B. 2008, 112, 5428-5438.

(44) Idrissi, A.; Hantal, G.; Jedlovszky, P. Properties of the Liquid-Vapor Interface of Acetone-Methanol Mixtures, as Seen from Computer Simulation and ITIM Surface Analysis. Phys. Chem. Chem. Phys. 2015, 17, 8913-8926. 


\section{Tables}

TABLE 1. Interaction Parameters of the Molecular Models Used.

\begin{tabular}{ccccc}
\hline \hline molecule & interaction site & $\sigma / \AA$ & $\varepsilon / \mathrm{kJ} \mathrm{mol}^{-1}$ & $q / \mathrm{e}$ \\
\hline $\mathrm{CCl}_{4}{ }^{\mathrm{a}}$ & $\mathrm{C}$ & 3.800 & 0.2093 & 0.248 \\
& $\mathrm{Cl}$ & 3.470 & 1.1137 & -0.062 \\
acetone $^{\mathrm{b}}$ & $\mathrm{CH}_{3}$ & 3.790 & 0.8144 & 0.000 \\
& $\mathrm{C}$ & 3.820 & 0.3324 & 0.424 \\
& $\mathrm{O}$ & 3.050 & 0.6565 & -0.424 \\
acetonitrile $^{\mathrm{c}}$ & $\mathrm{H}$ & 2.200 & 0.0835 & 0.201 \\
& $\mathrm{C}\left(\mathrm{H}_{3}\right)$ & 3.000 & 0.4177 & -0.577 \\
& $\mathrm{C}(\equiv \mathrm{N})$ & 3.400 & 0.4177 & 0.488 \\
methanol $^{\mathrm{d}}$ & $\mathrm{N}$ & 3.300 & 0.4177 & -0.514 \\
& $\mathrm{CH}$ & 3.601 & 1.0168 & 0.266 \\
& $\mathrm{O}$ & 3.176 & 0.5519 & -0.674 \\
water $^{\mathrm{e}}$ & $\mathrm{H}$ & - & - & 0.408 \\
& $\mathrm{O}$ & 3.166 & 0.6502 & -0.820 \\
\hline \hline
\end{tabular}

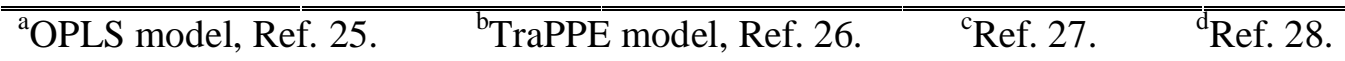

${ }^{\text {e}}$ SPC/E model, Ref. 29. 


\section{Figure legend}

Figure 1. Schematic structure of the five molecules considered in this study. C, Cl, O, N, and $\mathrm{H}$ atoms are marked by grey, light blue, red, dark blue, and white colors, respectively. $\mathrm{H}$ atoms are only shown when the molecular model used treats them explicitly.

Figure 2. Equilibrium snapshot of the surface portion of the five interfacial systems simulated. Molecules belonging to the first, second, third, fourth and fifth molecular layers are marked by blue, red, green, magenta, and brown colors, while those belonging to the bulk liquid phase (i.e. located beyond the fifth layer) and vapor phases by gray and yellow colors, respectively.

Figure 3. Non-intrinsic (i.e., relative to the basic simulation box) profile of the tangential pressure (black solid lines) at the liquid-vapor interface of $\mathrm{CCl}_{4}$ (top panel), acetone (second panel), acetonitrile (third panel), methanol (fourth panel), and water (bottom panel), as obtained from our computer simulations. The contributions given by the first (red squares), second (green circles), third (blue up triangles), fourth (light blue down triangles) and fifth (magenta open diamonds) molecular layers beneath the liquid surface to these profiles are also indicated. The insets show the structured part of the profiles on a magnified scale.

Figure 4. Intrinsic (i.e., relative to the capillary wave corrugated liquid surface) profile of the tangential pressure (black solid lines) at the liquid-vapor interface of $\mathrm{CCl}_{4}$ (top panel), acetone (second panel), acetonitrile (third panel), methanol (fourth panel), and water (bottom panel), as obtained from our computer simulations. The contributions given by the first (red squares), second (green circles), third (blue up triangles), fourth (light blue down triangles) and fifth (magenta open diamonds) molecular layers beneath the liquid surface to these profiles are also indicated.

Figure 5. Net contribution of the first four molecular layers beneath the liquid surface to the value of the surface tension, as obtained at the liquid-vapor interface of $\mathrm{CCl}_{4}$ (top panel), acetone (second panel), acetonitrile (third panel), methanol (fourth panel), and water (bottom panel). The corresponding values are also shown numerically. 
Figure 1.

Sega et al.
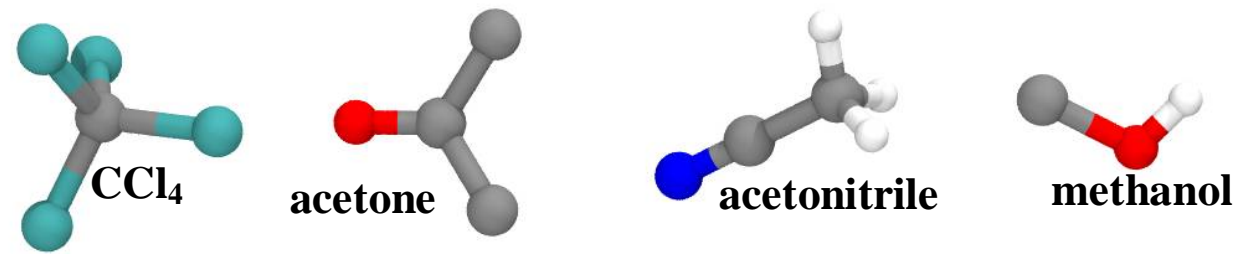

water 
Figure 2.

Sega et al.
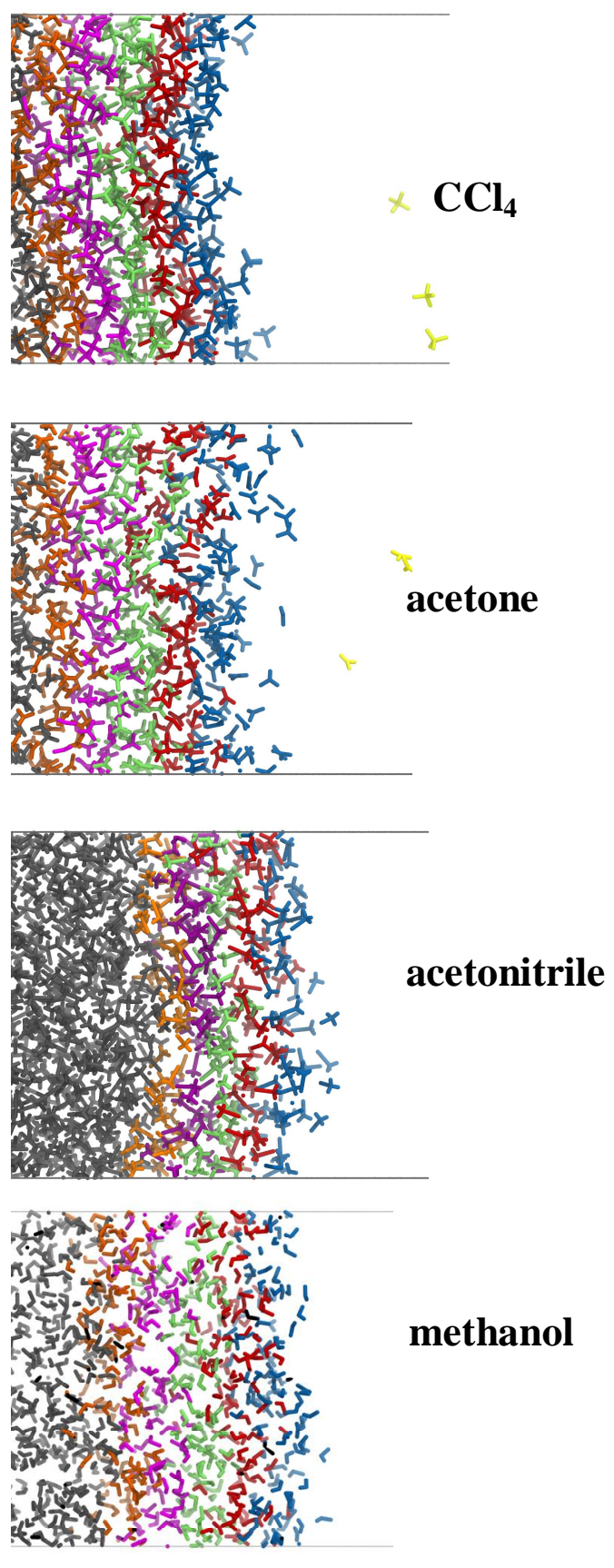

methanol

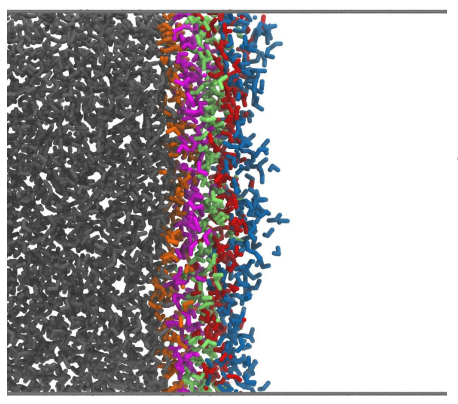

water 
Figure 3.

Sega et al.

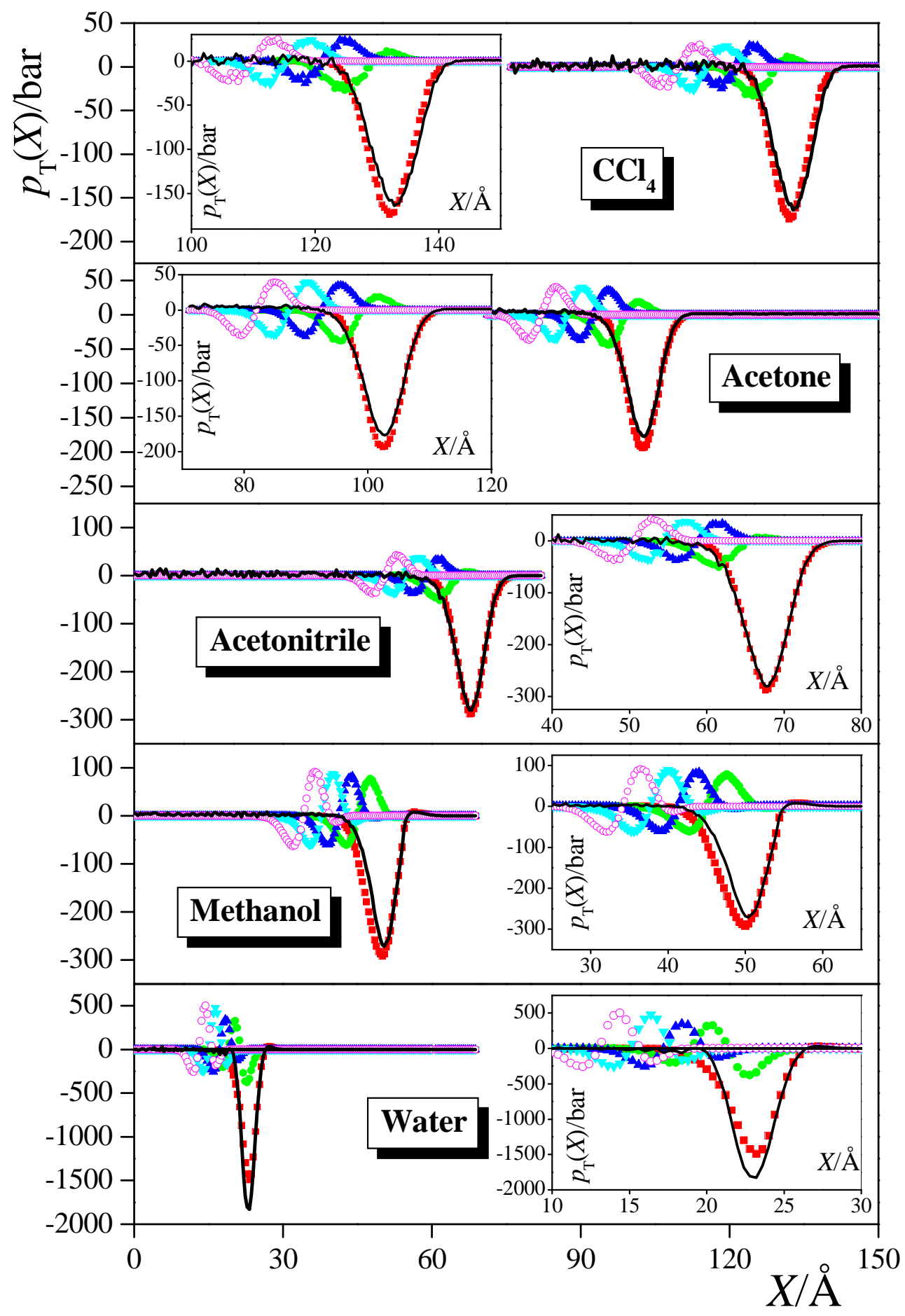


Figure 4.

Sega et al.

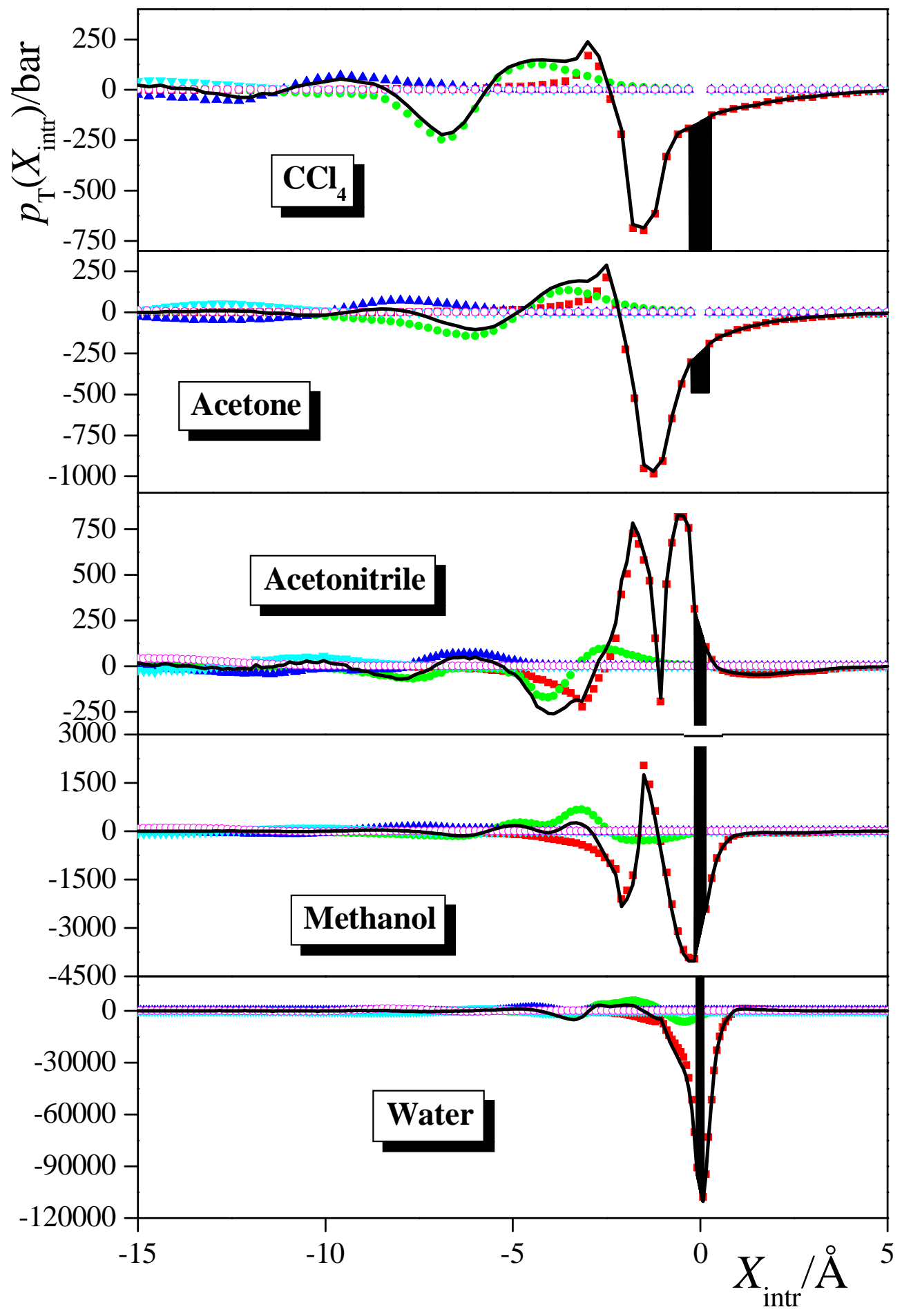


Figure 5.

Sega et al.

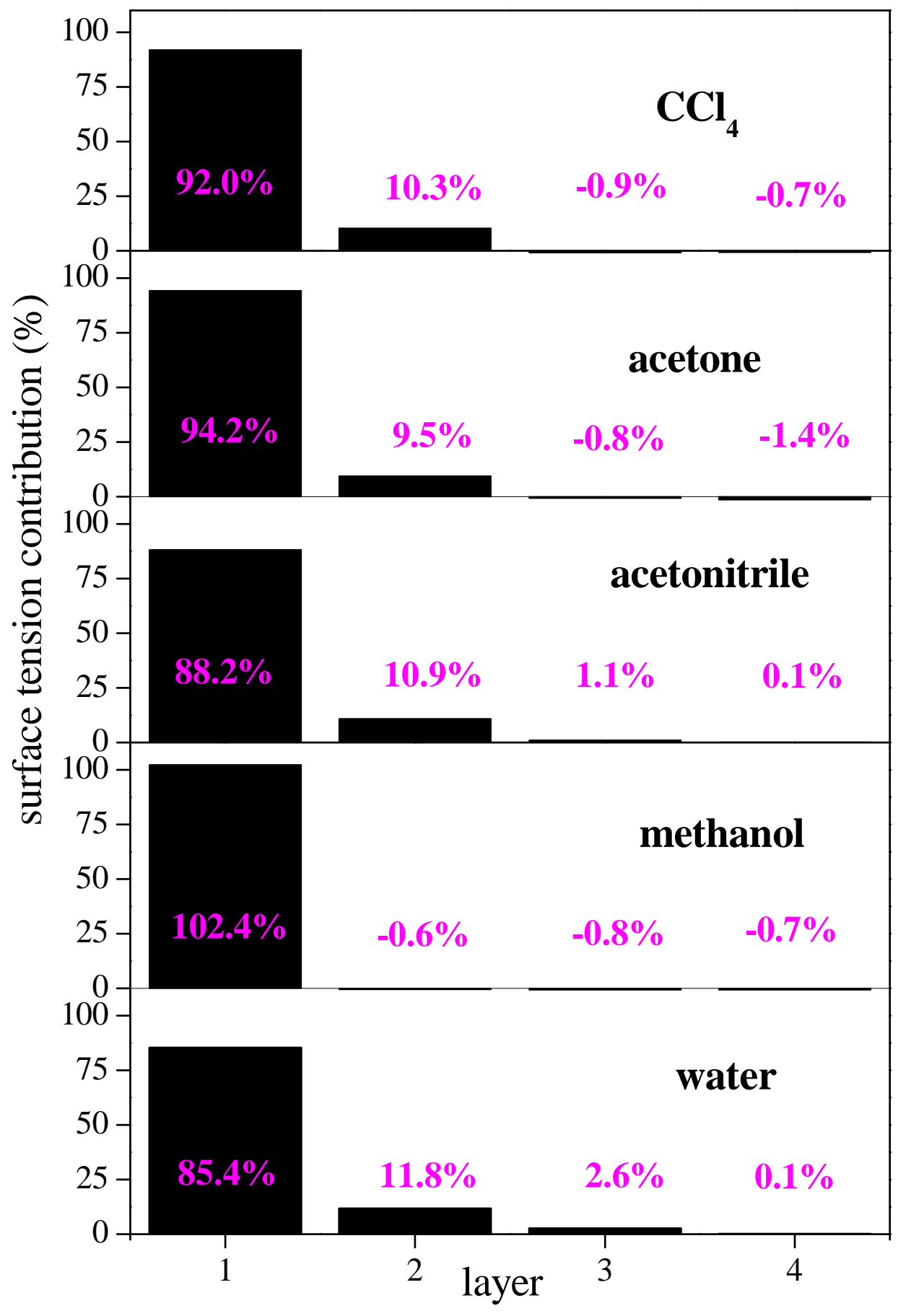


Table of Contents Graphics:

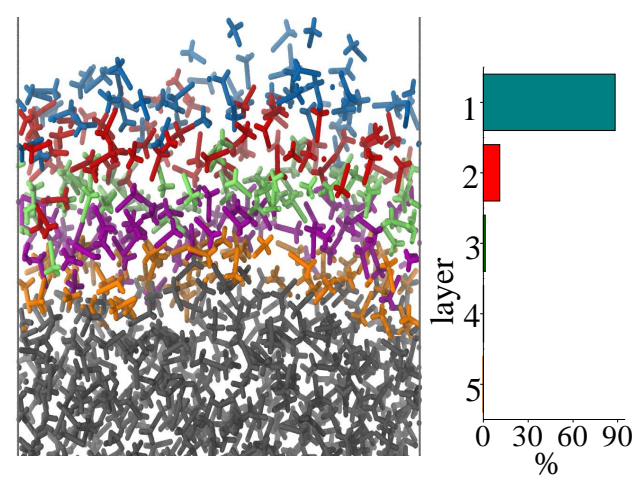

